\title{
Terapia Assistida por Animais como recurso fisioterapêutico para idosos institucionalizados
}

\author{
Animal-assisted Therapy as a physical therapy resource for institutionalized elderly
}

\author{
Fernanda Cechetti ${ }^{1,2} \bowtie$, Aline de Souza Pagnussat ${ }^{2}$, Karenina Elisa Marin ${ }^{3}$, Priscila Bertuol ${ }^{3}$, \\ Flávia Zambom Todero ${ }^{3}$, Suelen Antônia de Oliveira Ballardim ${ }^{3}$ \\ ${ }^{1}$ Departamento de Fisioterapia da Universidade Federal de Ciências da Saúde de Porto Alegre. Porto Alegre, RS. \\ 2 Programa de Pós-Graduação em Ciências da Reabilitação e Programa de Pós-Graduação em Ciências da Saúde da Universidade Federal de \\ Ciências da Saúde de Porto Alegre. Porto Alegre, RS. \\ ${ }^{3}$ Curso de Fisioterapia da Universidade de Caxias do Sul. Caxias do Sul, RS.
}

\section{RESUMO}

Objetivos: Avaliar os efeitos da Terapia Assistida por Animais em relação à marcha e ao equilíbrio em idosos institucionalizados.

Métodos: Este estudo caracteriza-se como um ensaio clínico não controlado, do tipo antes e depois. O estudo foi realizado na instituição Lar da Velhice São Francisco de Assis, localizado na cidade de Caxias do Sul, Rio Grande do Sul. Foram selecionados idosos voluntários, de ambos os sexos, sendo excluídos os que apresentassem alguma alteração osteomuscular, neurológica ou cognitiva que pudesse interferir nos resultados. Foram utilizados três cães, devidamente treinados por um profissional de adestramento e atendendo a critérios específicos para Terapia Assistida por Animais. O grupo realizou 10 sessões da terapia em quatro semanas. Para análise da marcha e equilíbrio dos idosos foram utilizados os seguintes testes: Escala de Equilíbrio de Berg, teste de Equilíbrio de Tinetti, teste de Alcance Funcional e teste de Caminhada de Seis Metros. Os testes foram aplicados antes e depois das sessões de Terapia Assistida por Animais.

Resultados: Foram incluídos no estudo nove idosos, com idades entre 68 e 79 anos. Em relação ao equilíbrio e controle postural os indivíduos apresentaram uma melhora significativa nos valores pós-tratamento quando comparados aos do pré-tratamento, pela Escala de Berg $(\mathrm{z}=2,26$ $\mathrm{p}=0,02)$ e pelo teste de Alcance Funcional $(\mathrm{z}=2,2 \mathrm{p}=0,02)$. Na avaliação dos aspectos da marcha, no teste de Tinetti a média do escore préterapia foi de $7,9 \pm 2,8$ e pós-terapia $9,2 \pm 1,6(z=2,02 p=0,04)$. O teste de Caminhada revelou uma significativa diminuição no tempo gasto para completar o percurso de seis metros após a terapia. A média do tempo antes da terapia foi de $20,2 \pm 19$ segundos e após a terapia foi de $9,2 \pm 7$ segundos $(\mathrm{z}=2,47 \mathrm{p}=0,01)(\mathrm{z}=2,47 \mathrm{p}=0,01)$.

Conclusões: Encontrou-se uma melhora significativa em ambos os parâmetros avaliados, sugerindo resultados relevantes após aplicação da Terapia Assistida por Animais em relação à marcha e ao equilíbrio de idosos institucionalizados.

DESCRITORES: terapia assistida por animais; idosos; institucionalização; fisioterapia.

\section{ABSTRACT}

Aims: To evaluate the effects of Animal-assisted Therapy on the gait and balance of institutionalized elderly.

Methods: This is a non-controlled before-after clinical trial. The study was conducted at "Lar da Velhice São Francisco de Assis,", a nursing home located in Caxias do Sul, Rio Grande do Sul, Brazil. Elderly volunteers of both sexes were selected, and those with any musculoskeletal, neurological, or cognitive conditions that could interfere with the results were excluded. Three dogs that were properly trained by a professional trainer and met the specific criteria for animal-assisted therapy were used. The group performed 10 therapy sessions in four weeks. The following tests were used for assessing the gait and balance of the elderly participants: Berg Balance scale, Tinetti Balance test, Functional Reach test, and Six-Meter-Walk test. The tests were applied before and after animal-assisted therapy.

Results: The study included nine subjects, aged 68 to 79 years. Regarding balance and postural control, individuals showed a significant improvement in post-treatment outcome when compared to pre-treatment, according to the Berg Balance scale $(\mathrm{z}=2.26 \mathrm{p}=0.02)$ and Functional Reach test $(\mathrm{z}=2.2 \mathrm{p}=0.02)$. In the gait assessment, the Tinetti test score averaged $7.9 \pm 2.8$ before therapy and $9.2 \pm 1.6$ after therapy $(\mathrm{z}=2.02$ $\mathrm{p}=0.04)$. The Six-Meter-Walk test after therapy revealed a significant decrease in the time necessary to complete the six-meter course. The average time was $20.2 \pm 19$ seconds prior to therapy and $9.2 \pm 7$ seconds after therapy $(\mathrm{z}=2.47 \mathrm{p}=0.01)$.

Conclusions: Both assessed parameters showed significant improvement, suggesting important outcomes regarding gait and balance after the use of Animal-assisted Therapy in institutionalized elderly individuals.

KEY WORDS: animal assisted therapy; elderly; institutionalization; physical therapy specialty. 
Abreviaturas: TAA, terapia assistida por animais; MEEM, Mini Exame do Estado Mental.

\section{INTRODUÇÃO}

O envelhecimento populacional é um fenômeno que vem acontecendo mundialmente. No Brasil, segundo último Censo realizado pelo IBGE em 2010, os idosos passaram a representar $10,8 \%$ da população, ou seja, mais de 20,5 milhões de brasileiros possuem mais de 60 anos, o que representa um incremento de $400 \%$ comparando ao índice anterior [1]. Proporcional ao acréscimo do número, tem aumentado também a preocupação com o bem estar, com a manutenção de um estilo de vida independente e com a qualidade de vida dos idosos, incluindo os que vivem em instituições de longa permanência $[2,3]$. As dificuldades enfrentadas pelos idosos na realização das atividades da vida diária são potencializadas nos institucionalizados $[3,4]$.

Dentre as principais alterações fisiológicas decorrentes do envelhecimento, situam-se a diminuição da capacidade de realizar uma marcha normal e a dificuldade em manter o equilíbrio. Falhas nos sistemas nervoso e musculoesquelético podem predispor a quedas, inclusive com risco de resultar em um evento fatal [5]. Ao adquirir hábitos de vida saudáveis, através de uma escolha pessoal ou por oportunidades oferecidas, essas alterações podem ser minimizadas, oportunizando ao idoso maior integração social, segurança e bem-estar [6].

Uma das estratégias existentes para a manutenção da independência dos idosos é a Terapia Assistida por Animais (TAA), que envolve a interação entre homem e animal em um processo terapêutico formal, guiado por profissionais da saúde qualificados, seguindo uma programação com objetivos claros e dirigidos. Os resultados esperados da TAA incluem promoção da saúde física, social e emocional, assim como melhora nas funções cognitivas [7].

A TAA teve origem em 1792, no York Retreat, Inglaterra, uma instituição filantrópica para doentes mentais, onde os pacientes tinham permissão para cuidar de animais como reforço positivo. Em 1942, nos Estados Unidos, terapeutas constataram o benefício do uso da TAA em pacientes com distúrbios físicos e mentais. No Brasil, a partir de 1955, a médica psiquiatra Nise da Silveira iniciou o processo com cães e gatos para doentes mentais, sendo considerada pioneira da TAA no País. Em 2000, com o objetivo de melhorar a qualidade de vida, o bem estar, a integridade e o respeito ao idoso, foi fundada a Organização Brasileira de Interação Homem-Animal Cão Coração, que promove o Projeto Cão do Idoso [8].

Na década de 1990 são implantados no Brasil os primeiros centros de atendimento de TAA, onde cães têm sido usados como facilitadores por profissionais de diversas áreas da saúde. Na Fisioterapia, a TAA aumenta a motivação dos pacientes durante as sessões, sendo o cão o agente estimulador e mediador das ações propostas durante o tratamento [9].

Entretanto, existem poucos estudos demonstrando os benefícios da TAA dentro da área da Fisioterapia. Assim, o objetivo principal deste estudo foi identificar os efeitos da TAA em relação ao equilíbrio e à marcha, em idosos institucionalizados.

\section{MÉTODOS}

Este trabalho caracterizou-se como um ensaio clínico não controlado, do tipo antes e depois. A pesquisa foi realizada no Lar São Francisco de Assis, uma instituição pública que abriga idosos em Caixas do Sul, cidade localizada na região serrana do Rio Grande do Sul.

Foram incluídos no estudo voluntários de ambos os sexos, que se enquadravam nos seguintes critérios: idade acima de 60 anos; indivíduos aparentemente sadios, que apresentavam apenas sinais e sintomas fisiológicos do envelhecimento; sem história de alergia a pelo do animal; que se posicionaram a favor da interação com animais; e que não estavam realizando tratamento fisioterapêutico. Foram excluídos do estudo idosos que, após uma primeira avaliação, apresentassem alguma alteração neurológica ou osteomuscular, ou algum quadro demencial instalado, que pudessem interferir nos resultados. Os idosos passaram por uma avaliação física simples realizada pelos próprios pesquisadores a fim de detectar alterações, e por uma avaliação cognitiva.

Para a avaliação cognitiva, foi realizado o Teste Mini Exame do Estado Mental (MEEM), um instrumento de rastreamento de comprometimento cognitivo. Os pontos de corte utilizados para o MEEM foram: 20 pontos para analfabetos, 25 pontos para um a quatro anos de estudo, 26,5 pontos para cinco a oito anos de estudo, 28 pontos para nove a onze anos de estudo e 29 pontos para mais de onze anos de estudo. Foram excluídos os idosos que apresentassem valores inferiores ao ponto de corte conforme os respectivos anos de estudo.

Para a TAA, foram utilizados três cães de raças distintas: um Golden Retriever macho, um Labrador 
fêmea e um Pastor Alemão fêmea, todos devidamente treinados por um profissional de adestramento e atendendo a critérios específicos para TAA.

Os exercícios com os cães incluíram: arremessar uma bola ao cão; deambular com o cão por diferentes terrenos e ultrapassando diversos obstáculos; com o paciente sentado, estender os joelhos para que o animal consiga passar por baixo de suas pernas, sem apoiar-se quando possível; em pé apoiado na parede, flexionar o joelho a $90^{\circ}$ para que o animal possa passar por baixo de sua perna, e logo após flexionar a outra perna e acariciar o animal com o animal posicionado atrás; e realizar exercício de senta-levanta, imitando o cão à sua frente, sendo que o comando era dado pelo adestrador através de sinais. Esses exercícios objetivam melhora do controle postural e melhor desempenho no âmbito da marcha, principalmente em relação à velocidade $\mathrm{e}$ à realização correta das fases da marcha. O número de repetições e a dificuldade dos exercícios foram sendo aumentados conforme a progressão individual de cada participante.

O tratamento consistiu de 10 sessões de TAA em quatro semanas, distribuídas de forma a haver uma diminuição gradual da frequência, a fim de ocorrer um desapego lento em relação ao animal por parte do idoso. Assim, na primeira semana foram realizadas quatro sessões, na segunda três sessões, na terceira duas sessões e por fim uma sessão na quarta e última semana, com duração de 45 minutos cada. As atividades foram realizadas em grupo, e todos os dias de intervenção foram agendados previamente com os participantes. Além disso, as atividades eram ministradas sempre pelos mesmos profissionais envolvidos no estudo e os objetivos específicos do tratamento eram planejados para cada sessão.

Os seguintes testes foram realizados antes e após a intervenção: Escala de Equilíbrio de Berg, teste de Equilíbrio de Tinetti, teste de Alcance Funcional e teste de Caminhada de Seis Metros.

A Escala de Equilíbrio de Berg avalia o desempenho do equilíbrio funcional em 14 itens comuns à vida diária. Quanto maior a pontuação melhor o equilíbrio, sendo que a pontuação máxima chega a 56 pontos.

$\mathrm{O}$ teste de Equilíbrio de Tinetti classifica aspectos da marcha, como velocidade, distância do passo e simetria sendo o escore máximo de 12 pontos, atingido quando o desempenho é o melhor possível [10].

O teste de Alcance Funcional, elaborado por Duncan et al. [11], é utilizado para identificar alterações dinâmicas do controle postural. O resultado é fornecido pela média da medida do comprimento alcançado após três tentativas. Deslocamentos me- nores do que $15 \mathrm{~cm}$ indicam fragilidade e risco de quedas [12].

O teste de Caminhada de Seis Metros foi realizado cronometrando o tempo que cada idoso precisava caminhar para percorrer $6 \mathrm{~m}$ em um corredor. Quanto menor o tempo para realizar o percurso, significa melhor desempenho do participante, indicando melhora na capacidade de marcha.

O projeto foi aprovado pelo Comitê de Ética em Pesquisa da Fundação Universidade de Caxias do Sul sob número 338/10. Todos os voluntários assinaram o termo de consentimento livre e esclarecido, de acordo com a Resolução 459/2012 do Conselho Nacional de Saúde.

Os resultados obtidos foram codificados, revisados e digitados em um banco de dados construído no programa IBM SPSS Statistics 18.0. Pelo teste de Shapiro-Wilk os dados foram considerados nãoparamétricos e analisados por meio do teste de Wilcoxon. Foi considerada significância estatística um $\mathrm{p}<0,05$.

\section{RESULTADOS}

Foram selecionados inicialmente 17 idosos que preenchiam os critérios de inclusão. Após os testes iniciais, foram excluídos quatro por possuírem alguma alteração osteomuscular ou neurológica associada e quatro por apresentarem comprometimento cognitivo segundo o MEEM. Portanto, foram incluídos no estudo nove idosos voluntários, com idades entre 68 e 79 anos (média de 73,1 $\pm 4,3$ anos).

Pela Escala de Equilíbrio de Berg, a maioria dos indivíduos mostrou aumento da pontuação no teste pós intervenção, demonstrando melhora no equilíbrio. A pontuação média foi de $44 \pm 16$ antes da TAA e $52 \pm 5$ após a TAA $(\mathrm{z}=2,26 \mathrm{p}=0,02)$ (Tabela 1).

A análise dos escores do teste de Tinetti mostrou média pré-TAA de $7,9 \pm 2,8$ e pós-TAA de $9,2 \pm 1,6$ $(\mathrm{z}=2,02 \mathrm{p}=0,04)$, indicando melhora na velocidade, distância do passo e simetria da marcha após o tratamento (Tabela 1).

Os resultados referentes ao teste de Alcance Funcional mostraram significativo aumento dos valores após o tratamento. A média foi de $16 \pm 7$ pontos antes e $18 \pm 7$ pontos após $(\mathrm{z}=2,2 \mathrm{p}=0,02)$ (Tabela 1$)$.

$\mathrm{O}$ teste de Caminhada de Seis Metros evidenciou uma significativa diminuição no tempo gasto pelos idosos para completar os $6 \mathrm{~m}$ após a terapia. A média do tempo de realização do Teste antes da TAA foi de $20,2 \pm 19$ segundos e após a TAA foi de $9,2 \pm 7$ segundos $(\mathrm{z}=2,47 \mathrm{p}=0,01)$ (Tabela 1). 
Tabela 1. Demonstração dos valores individuais e das médias da pontuação obtida nos diversos testes aplicados a idosos institucionalizados, antes e após 10 sessões de Terapia Assistida por Animais, no decorrer de quatro semanas.

\begin{tabular}{|c|c|c|c|c|c|c|c|c|c|}
\hline \multirow[t]{2}{*}{$\begin{array}{l}\text { Participantes } \\
\quad \mathrm{n}=9\end{array}$} & \multirow[t]{2}{*}{$\begin{array}{l}\text { Idade } \\
\text { (anos) }\end{array}$} & \multicolumn{2}{|c|}{$\begin{array}{l}\text { Teste de Equilíbrio de Berg } \\
\text { (pontuação) }\end{array}$} & \multicolumn{2}{|c|}{$\begin{array}{l}\text { Teste de Equilíbrio deTinetti } \\
\text { (escore) }\end{array}$} & \multicolumn{2}{|c|}{$\begin{array}{l}\text { Teste de Alcance Funcional } \\
\text { (pontuação) }\end{array}$} & \multicolumn{2}{|c|}{$\begin{array}{l}\text { Teste de Caminhada } \\
\text { de Seis Metros } \\
\text { (segundos*) }\end{array}$} \\
\hline & & Pré-TAA & Pós-TAA & Pré-TAA & Pós-TAA & Pré-TAA & Pós-TAA & Pré-TAA & Pós-TAA \\
\hline 1 & 72 & 48 & 56 & 5 & 7 & 21 & 22 & 23 & 7 \\
\hline 2 & 70 & 5 & 42 & 7 & 9 & 22 & 24 & 70 & 28 \\
\hline 3 & 71 & 26 & 44 & 8 & 9 & 25 & 27 & 25 & 13 \\
\hline 4 & 68 & 53 & 53 & 10 & 10 & 27 & 27 & 11 & 7 \\
\hline 5 & 79 & 45 & 52 & 10 & 11 & 10 & 11 & 13 & 8 \\
\hline 6 & 72 & 54 & 56 & 11 & 12 & 17 & 17 & 17 & 4 \\
\hline 7 & 69 & 54 & 56 & 5 & 7 & 10 & 13 & 3 & 3 \\
\hline 8 & 78 & 56 & 56 & 8 & 9 & 6 & 9 & 10 & 5 \\
\hline 9 & 79 & 49 & 52 & 7 & 9 & 18 & 21 & 10 & 8 \\
\hline Média „DP & $73,1 \pm 4,3$ & $44 \pm 16$ & $52 \pm 5$ & $7,9 \pm 2$ & $9,2 \pm 1,6$ & $16 \pm 7$ & $18 \pm 7$ & $20,2 \pm 19$ & $9,2 \pm 7$ \\
\hline Valor de $\mathrm{p}^{+}$ & - & \multicolumn{2}{|c|}{$\mathrm{p}=0,02$} & \multicolumn{2}{|c|}{$p=0,04$} & \multicolumn{2}{|c|}{$p=0,02$} & \multicolumn{2}{|c|}{$p=0,01$} \\
\hline
\end{tabular}

Pré TAA, antes da Terapia Assistida por Animais; pós-TAA, após a Terapia Assistida por Animais; DP, desvio padrão.

* Tempo dispendido para perfazer o percurso de 6 metros.

+ Teste de Wilcoxon. Comparação entre as médias pré e pós-terapia.

\section{DISCUSSÃO}

Os resultados obtidos no presente estudo demonstram claramente a melhora motora apresentada pelos idosos, após as sessões de fisioterapia utilizando o cão como instrumento facilitador. Observou-se melhora em todos os parâmetros estudados: equilíbrio, tempo de caminhada, distância do passo, simetria e dinâmica do controle postural.

$\mathrm{Na}$ literatura já foram observados diversos benefícios da terapia que utiliza o cão como instrumento no tratamento de idosos. O primeiro deles é o estímulo à interação social. Os idosos veem os cães como seus amigos especiais, membros importantes da família. $\mathrm{O}$ processo de envelhecimento fisiológico traz consigo diversas alterações na vida dos idosos, incluindo dificuldade em interagir com outras pessoas. Com ajuda dos cães, fica mais fácil conquistar confiança e formar vínculos. O segundo benefício é a motivação, pois as atividades lúdicas com o cão estimulam o interesse no contato interpessoal, auxiliando no tratamento. O terceiro benefício é a melhora da capacidade motora, cognitiva e sensorial, sendo de grande ajuda na fisioterapia, pois os animais fazem a ponte entre o idoso e o terapeuta, que assim pode atuar com mais eficiência e rapidez [7].

Um fator importante do envelhecimento é que ele compromete a manutenção do equilíbrio corporal e a capacidade de modificação dos reflexos adaptativos, principalmente por afetar a capacidade do sistema nervoso central, em especial das regiões envolvidas nesse processo: sistemas vestibular, visual e proprioceptivo. Isso resulta diretamente no aparecimento de tonturas e desequilíbrio nos longevos [13], sendo considerado um dos fatores limitantes da qualidade de vida dos idosos. O comprometimento desse sistema tem como consequências a dificuldade de locomoção e o risco de quedas, que por sua vez podem resultar em fraturas, deixando os idosos acamados por dias ou meses [14]. As quedas são responsáveis por $70 \%$ das mortes acidentais em pessoas com mais de 75 anos [15].

A TAA é utilizada na Fisioterapia como uma ferramenta de trabalho, com grande vantagem de adaptação a diferentes técnicas que o profissional possa vir a realizar em seus pacientes. A presença dos cães ajuda a tornar a fisioterapia mais agradável e, além disso, dá ao idoso um companheiro que o motiva o tempo todo e faz graça toda vez que ele realiza o movimento correto [16]. Estudos citam que o estímulo proporcionado pelo tato, somado à presença do animal, recupera a autoestima e a sensibilidade global dos pacientes [17].

A literatura destaca ainda outros benefícios específicos da TAA, como aperfeiçoamento das habilidades motoras finas; melhora do equilíbrio ao sustentar-se; melhora da adesão ao tratamento; aumentada interação verbal entre os membros do grupo; melhora das habilidades de atenção; aumento da autoestima; redução da ansiedade; diminuição da solidão; aperfeiçoamento do conhecimento dos conceitos de tamanho e cor; melhorada interação com a equipe de saúde; e motivação para o envolvimento em 
atividades de grupo. Por sua vez, os animais tornamse importantes na comunicação entre terapeutas e pacientes $[18,19]$.

Todos esses efeitos contribuem para que a mobilidade dos idosos também apresente melhores resultados, amenizando os déficits na marcha desses indivíduos, causados pelo processo fisiológico do envelhecimento. Tanto o teste de Tinetti como o teste de Caminhada de Seis Metros aplicados neste estudo demonstraram resultados positivos e significativos em relação às sessões de fisioterapia realizadas com auxílio de cães. O mesmo ocorreu com o equilíbrio destes idosos avaliados pela Escala de Berg e teste de Alcance Funcional. Como principal limitação deste estudo, citamos o baixo número de participantes, contudo, mesmo assim, os resultados foram significativos.

Estudo realizado por Mashimo e Caromano [20] mostrou que a maioria dos idosos apresenta a mobilidade do tronco diminuída durante a marcha. Esse aspecto foi determinado pelo grande número de idosos que apresentavam diminuição na rotação das cinturas pélvica e escapular, caracterizando a "marcha em bloco". Naquele estudo, $61 \%$ dos idosos apresentaram diminuição na rotação de cintura pélvica, $9 \%$ não realizaram a rotação e $30 \%$ realizaram a rotação normal. Em relação à rotação de cintura escapular, $70 \%$ dos idosos apresentaram rotação diminuída, $26 \%$ não realizaram a rotação e apenas $4 \%$ da amostra apresentou a rotação normal [20].

Essa diminuição geral de mobilidade pode ser amenizada se o idoso realizar alguma atividade que o tire da rotina do dia-a-dia e, principalmente, que lhe cause bem estar. Autores citam que a convivência com animais proporciona ao paciente alegria, troca de afeto, segurança, motivação e socialização, além melhorar a saúde física pelo simples fato de levar o cão para passear ou fazer suas necessidades fisiológicas todos os dias. A simples presença de um animal de estimação pode fazer com que ocorra uma melhora da mobilidade e, consequentemente, do equilíbrio e da marcha, do sentimento de segurança e da atenção, além de estimular a motivação e diminuir a ansiedade [16,21].

Para reforçar esta afirmação em relação à melhora da mobilidade geral dos idosos, um grande estudo realizado por Mossello et al. [22] aplicaram a TAA durante três semanas em um grupo de idosos com mal de Alzheimer na fase avançada. Dentre os principais resultados encontrados, houve significativa melhora da atividade motora, diminuição da ansiedade e aumento de emoções positivas. Outro achado muito interessante foi que as melhoras encontradas permaneciam até quatro horas após a sessão de terapia com os cães. Segundo esse grupo de pesquisadores, um dos principais fatores responsáveis pelo sucesso da terapia é a maneira pela qual o cão comunica-se com o idoso, ou seja, do tipo não verbal e sem pré-julgamentos. Além disso, juntamente com outros autores, reforçam que a abordagem terapêutica de tratamento para os idosos deve preconizar a estimulação ambiental multissensorial, algo naturalmente conquistado durante as sessões de TAA [22,23].

Atualmente, a TAA é vista como uma técnica em potencial, e, mesmo apresentando um caráter interdisciplinar, ainda é restrita a iniciativas particulares. Mesmo sendo indicada por profissionais de várias áreas da saúde, necessita de mais estudos para ser realmente validada e alcançar suficientes evidências científicas sobre a contribuição, tanto nos aspectos fisiológicos como psicológicos, do uso do animal como uma ferramenta terapêutica $[8,23,24]$.

Após realizada a análise dos dados obtidos neste estudo durante a atividade proposta e avaliados pelos testes utilizados, podemos concluir, não obstante o pequeno número amostral, que a TAA foi capaz de trazer resultados positivos em relação à melhora da marcha e do equilíbrio em idosos institucionalizados. Sendo assim, pode-se dizer que é possível realizar as intervenções de TAA em instituições de longa permanência para idosos, visando a aproveitar os seus benefícios em relação aos parâmetros analisados.

\section{NOTA}

Declaração de conflitos de interesse

Os autores declaram não haver conflitos de interesse relevantes ao conteúdo deste estudo.

\section{REFERÊNCIAS}

1. Instituto Brasileiro de Geografia e Estatística. Censo demográfico 2010 [Internet]. Rio de Janeiro: IBGE; 2014 [cited 2016]. Available from: http://www.ibge.gov.br./home/

2. Freitas Júnior P, Barela J. Alterações no funcionamento do sistema de controle postural em idosos. Uso da informação visual. Rev Port Cien Desp. 2006;6(1):94-105. 
3. Cechetti F, Reis C, Cabral T, Bett F, Rodrigues L, Bortolini R, Simioni F, Zanonato A, Schmitt G, Guazzelli N, Vigolo G, Lopes T, Finger F, Bolfe R, Roth F, Puhl L. Relação entre função cognitiva e capacidade funcional em idosos institucionalizados de Caxias do Sul/RS. Rev Fisioter Bras. 2011;12(5):347-52.

4. Vivan S, Argimon IIL. Estrategias de enfrentamento, dificuldades funcionais e fatores associados em idosos institucionalizados. Cad Saúde Públ. 2009;25(2):436-44. http://dx.doi.org/10.1590/S0102-311X2009000200022

5. Swift CG. Falls in late life and their consequences: implementing effective services. BMJ. 2001 Apr 7;322(7290):855-7. http://dx.doi. org/10.1136/bmj.322.7290.855

6. Farias RG, Santos SMA. Influência dos determinantes do envelhecimento ativo entre idosos mais idosos. Texto Contexto Enferm. 2012;21(1):167-76. http://dx.doi.org/10.1590/S0104-07072012000100019

7. Dotti J. Terapia e animais. São Paulo: PC Editorial; 2005.

8. Martins MF. Zooterapia ou terapia assistida por animais (TAA). Rev Nosso Clínico. 2004;40:22-6.

9. Vaccari AMH, Almeida FA. A importância da visita de animais de estimação na recuperação de crianças hospitalizadas. Einstein. 2007;5(2):111-6.

10. Tinett, M.E. Performance-oriented assessment of mobility problems in elderly pacients. J Am Geriatric Soc. 1986;34(1):119-26. http://dx.doi.org/10.1111/j.1532-5415.1986.tb05480.x

11. Duncan PW, Weiner DK, Chandler J, Studenski S. Functional reach: a new clinical measure of balance. J Gerontol. 1990 Nov;45(6):M192-7. http://dx.doi.org/10.1093/geronj/45.6.M192

12. Figueiredo KMOB, Lima KC, Guerra RO. Instrumentos de avaliação de equilíbrio corporal em idosos. Rev Bras Cineantropom Desempenho Hum. 2007;9(1):408-13.

13. Ruwer SL, Rossi AG, Simon LF. Equilíbrio no idoso. Rev Bras Otorrin. 2005;71(3):298-303. http://dx.doi.org/10.1590/S003472992005000300006

14. Figliolino JAM, Morais TB, Berbel AM, Corso SD. Análise da influência do exercício físico em idosos com relação a equilíbrio, marcha e atividade de vida diária. Rev Bras Geriatr Gerontol. 2009;12(2):227-38. http://dx.doi.org/10.1590/1809-9823.2009.12026

15. Bittar RSM, Pedaline MEB, Bottino MA, Formigoni LG. Síndrome do desequilíbrio no idoso. Pró-fono. 2002;1(14):119-28.

16. Pereira MJF, Pereira L, Ferreira ML. Os benefícios da terapia assistida por animais: uma revisão bibliográfica. Ciênc Saúde Colet. 2007;4(14):62-6.

17. SanJoaquín MPZ. Terapia asistida por animales de compa-ía. Bienestar para el ser humano. Rev Centro Salud. 2002;10(3):143-49.

18. Allen K. Dog ownwership and control of borderline hypertension: a control randomized trial. Bufalo: Millard Fillmore Hospital; 2001.

19. Fila D. The significance of companion animals to a geriatric vascular case study. Holistic Nurs Prac. 1991;5(2):19-25. http://dx.doi. org/10.1097/00004650-199101000-00005

20. Mashimo AM, Caromano FA. A marcha em idosos saudáveis. Arq Ciênc Saúde Unipar. 2002;2(11):117-21.

21. Kobayashi CT, Ushiyama ST, Fakih FT, Robles R, Carneiro IA, Carmagnani MIS. Desenvolvimento e implantação de terapia assistida por animais em hospital universitário. Rev Bras Enferm. 2009;62(4):632-6. http://dx.doi.org/10.1590/S0034-71672009000400024

22. Mossello E, Ridolfi A, Mello AM, Lorenzini G, Mugnai F, Piccini C, Barone D, Peruzzi A, Masotti G, Marchionni N. Animal-assisted activity and emotional status of patients with Alzheimer's disease in day care. Int Psychogeriatr. 2011 Aug;23(6):899-905. http://dx.doi. org/10.1017/S1041610211000226

23. Pecelin A, Furlan LA, Berbel AM, Lanuez FV. Influência da fisioterapia assistida por animais em relação á cognição de idosos - estudo de atualização. Conscientiae Saúde. 2007;6(2):235-40. http://dx.doi.org/10.5585/conssaude.v6i2.1075

24. Martin F, Farnum J. Animal-assisted therapy for children with pervasivedevelopmental disorders. West J Nurs Res. 2002 Oct;24(6): 657-70. http://dx.doi.org/10.1177/019394502320555403 